Division of Geological \& Geophysical Surveys

PRELIMINARY INTERPRETIVE REPORT 2003-3

\title{
PRINCIPAL FACTS FOR GRAVITY DATA COLLECTED IN THE SUSITNA BASIN AREA, SOUTHCENTRAL ALASKA
}

\author{
by \\ John F. Meyer Jr., and Peter L. Boggess \\ Alaska Division of Oil \& Gas, 550 W. $7^{\text {th }}$ Ave., Suite 800, Anchorage, AK 99501
}

December 2003

THIS REPORT HAS NOT BEEN REVIEWED FOR

TECHNICAL CONTENT (EXCEPT AS NOTED IN TEXT) OR FOR

CONFORMITY TO THE EDITORIAL STANDARDS OF DGGS.

Released by

STATE OF ALASKA

DEPARTMENT OF NATURAL RESOURCES

Division of Geological \& Geophysical Surveys

3354 College Rd.

Fairbanks, Alaska 99709-3707 


\section{INTRODUCTION}

The Susitna basin is a topographic lowland encompassing an area of approximately 4,700 square miles, bordered by the Alaska Range on the north, the Talkeetna Mountains on the east, the Tordrillo Mountains on the west and the Cook Inlet basin on the south. The Susitna basin is considered as a northern extension of the Cook Inlet basin, separated from it by the Castle Mountain fault, which is a major regional structural feature of southcentral Alaska.

The depositional history of the region started during the late Paleozoic and early Triassic when marine sediments were deposited throughout the area. This was followed by a time of deformation and uplift during the late Triassic, which resulted in the deposition of sediments in the Cook Inlet basin to the south. Uplift and erosion of the Alaska Range during the Cretaceous through Tertiary provided the material for a thick sequence of continental shelf deposits throughout the region that were composed of fine-grained sediments rich in organic material. During the Tertiary period, a repetitive cycle of vegetative growth and sediment deposition occurred depositing sediments along with conglomerates, sands and clays that created numerous peat layers which were buried, producing the present-day coal formations. The adjacent sands and gravels deposited in the region have become potential reservoirs for oil and gas in the area (AEIDC, 1974:41; Ryherd, 2003).

The structural style of the basin is a combination of graben and half-graben basement faulting with Tertiary sedimentary fill consisting of the same formations as found in the Cook Inlet basin. The sedimentary section ranges from about 2,000 feet thick just north of the Castle Mountain Fault to over 13,000 feet in the center of the basin, while south of the fault it is estimated to be at least 20,000 feet thick (Maynard, 1987; Ryherd, 2003). The Eocene-age West Foreland Formation and Oligocene age Hemlock Conglomerate reservoir rocks that are found to the south in the Cook Inlet basin appear to be missing in this basin. The presence of dry gas source rocks in the region, similar to those found in the Cook Inlet basin, and the apparent absence of equivalent oil-prone source rocks indicate that the potential for finding gas in the basin is much greater than for finding oil within the Tertiary section. Coal seams are thick and numerous in parts of the basin, and provide targets for methane drainage drilling as well as a source for gas to charge conventional sandstone reservoirs.

This basin has not been extensively explored although a number of oil and gas exploration wells have been drilled in the region. All of these wells were plugged and abandoned as dry holes, although some did have minor gas shows. There were also prominent coal beds in the lower part of some of the wells, suggesting a correlation with the coal-bearing formations in the Cook Inlet basin that produce natural gas. Taking this into account, the petroleum potential of the basin is thought to be low to moderate (Ryherd, 2003).

In order to help stimulate interest in this area for petroleum exploration, the Division of Oil and Gas (DO\&G) collected 120 additional gravity stations regionally and along profiles in the area during June and July of 2000. This survey was conducted in order to complement and extend the gravity data that is currently available from the U.S. Geological Survey (USGS). The gravity stations are located in the southeast corner of the Talkeetna and the northeast corner of the Tyonek 1:250,000 scale USGS topographic maps. The study area is bounded by $61^{\circ} 45^{\prime}$ to 
$62^{\circ} 30^{\prime} \mathrm{N}$. latitude and $150^{\circ} 00^{\prime}$ to $151^{\circ} 30^{\prime} \mathrm{W}$. longitude. Figure 2 represents a map of the study area showing the newly collected gravity stations in addition to the currently available USGS gravity stations.

\section{GRAVITY-DATA ACQUISITION AND REDUCTION}

A LaCoste and Romberg gravity meter (G507) was used to collect the new gravity station data. Conversion of the meter readings to milligals was made using factory calibration constants and a calibration factor determined by Dave Barnes of the USGS. During the field surveys, the gravity meter appeared to function properly, and a maximum drift of $0.09 \mathrm{mgal} / \mathrm{day}$ indicates there were no apparent tares in the data. The observed gravity values were based on an assumed linear drift between base station readings throughout the day.

Datum control for all of the gravity values was provided by the USGS Alaskan Gravity Base Station Network (Barnes, 1968; 1972) and was adjusted to the new absolute datum of the International Gravity Standardization Net 1971 (Morelli and others, 1974). A second-order base station, TLKN, was created at the new Talkeetna Railroad station as control for this survey. The observed gravity of the TLKN station was calculated based on multiple ties to the established TLKM base station. For the duration of the survey, this new station was reoccupied twice each day with survey loops limited to 10 hours or less.

Horizontal control was obtained using a Trimble Pathfinder Basic Plus portable Global Positioning System (GPS) unit and USGS topographic maps at a scale of 1:63,360. The station locations were located on USGS topographic maps in the field and digitized for comparison to the reduced GPS data. The GPS locations were processed using the Trimble GPS Pathfinder Office software and base station data obtained from the NOAA Continuously Operating Reference Stations (CORS) in Talkeetna and Anchorage. Standard processing techniques were applied to the GPS data by averaging the corrected data after applying differential corrections and selecting the best-corrected locations within the $68 \%$ confidence level. The accuracies for the corrected GPS locations were found to be \pm 8 feet, and in all but a few cases, were found to be of a higher accuracy, compared to the digitized locations. In a few cases, the GPS base station data did not collect enough information to get reasonable locations and the digitized values were used.

Vertical control was obtained using a Trimble Pathfinder Basic Plus portable GPS unit, American Paulin Model T-5 altimeters and USGS topographic maps at a scale of 1:63,360. The altimetry data was collected using three meters with the readings averaged at each station and corrected for diurnal barometric variations. Where feasible, the gravity stations were located at U.S. Coast and Geodetic Survey Vertical Angle Benchmarks (VABM) for comparison. Temperature and drift corrections were also applied and yielded elevations with an accuracy of \pm 30 feet. Elevations were also digitized from USGS topographic maps with an accuracy of \pm 50 feet and the GPS data was processed as described above and yielded data with an accuracy of \pm 14 feet. A comparison of the three elevations was made (Figure 1) and it was determined that for most of the stations, the GPS values provided the most accurate and consistent values for use in reducing the data. 

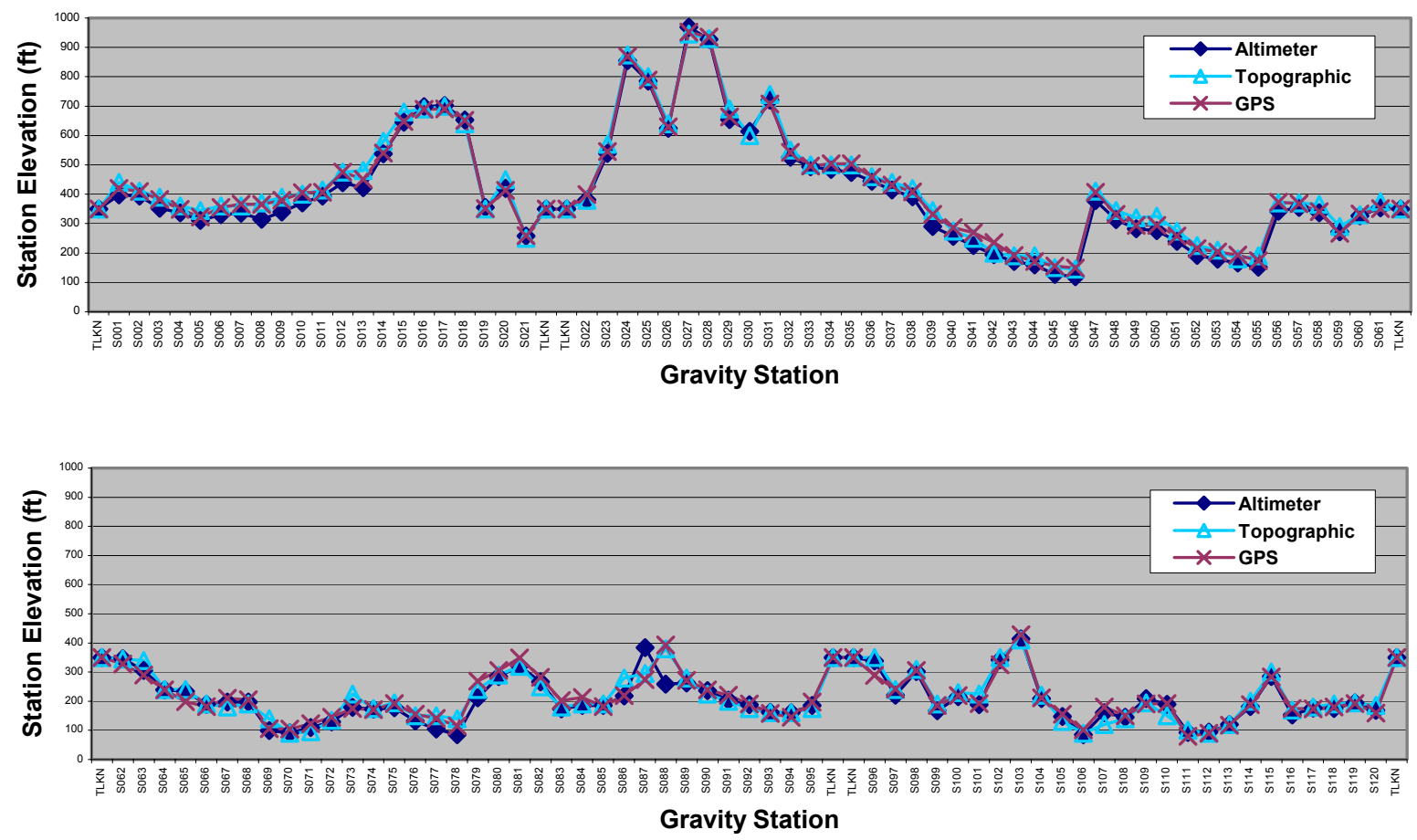

Figure 1. Graph comparing the altimetry, GPS and topographic station elevations.

Gravity reductions were run on all of the data (including the data obtained from the USGS) using standard techniques. Corrections for the variation of gravity with latitude at each station were computed based on the Geodetic Reference System 1967 (International Association of Geodesy, 1971) using the International Gravity Standardization Net 1971 gravity datum ( Morelli and others, 1974). The observed gravity values were calculated by adding the meter drift and earth-tide corrections to the meter readings converted to milligals. Free-air anomalies were calculated by subtracting the theoretical gravity from the observed gravity and adding a free-air correction. Simple Bouguer anomalies were calculated by subtracting the Bouguer correction from the free-air anomaly, calculated using a gravitational constant of $6.67 \times 10^{-11} \mathrm{~m}^{3} \mathrm{~kg}^{-1} \mathrm{~s}^{-2}$ and a standard density of $2.67 \mathrm{gm} / \mathrm{cc}$. Complete Bouguer anomalies were calculated by adding the terrain correction to the simple Bouguer anomaly and isostatic anomalies were calculated by adding the isostatic correction to the complete Bouguer anomaly.

Bob Morin of the USGS computed the terrain corrections for this data by using a computer program (Plouff, 1966, 1977; Godson and Plouff, 1988) and a digital terrain model. This program calculated the gravity effects of the surrounding terrain for each station from a radial distance of $0.39 \mathrm{~km}$ to a distance of $166.7 \mathrm{~km}$ using the standard Hammer technique (Hammer, 1939), in which average elevation estimates within zones surrounding the station are used to compute the gravity effect of each zone. The station elevations used for this correction were taken from the digitized USGS topographic maps at a scale of 1:63,360 in order to be consistent with the elevation model used for the terrain. No inner zone correction was applied due to the flat topography surrounding the stations. 
Bob Morin also processed these data with an isostatic reduction program (Jachens and Roberts, 1981) to compensate for the effects of crustal roots that buoyantly support topography. The isostatic reduction assumes an Airy-Heiskanen model with a density of topography above sea level of $2.6 \mathrm{gm} / \mathrm{cc}$ and a crustal thickness at sea level of $25 \mathrm{~km}$.

The locations of the gravity data collected in this survey as well as the data collected and available by the USGS can be seen in Figure 2. The data locations have been plotted on a topographic base with the new stations plotted in red while the USGS data are plotted in black. Figure 3 shows the contoured free-air anomaly values, Figure 4 shows the contoured complete Bouguer anomaly values and Figure 5 shows the contoured isostatic anomaly values. Table 1 lists the principal facts for the gravity stations collected during this survey.

\section{ACKNOWLEDGMENTS}

The authors gratefully acknowledge Tim Ryherd and Don Krouskop of the Division of Oil and Gas for reviewing the manuscript and providing many helpful suggestions. We also want to thank Kent Richter, Lana Ledlow \& Sunny Remmy with the Division of Oil and Gas for their assistance in collecting and reducing this data and Bob Morin with the USGS for computing all of the terrain and isostatic corrections.

\section{REFERENCES CITED}

AEIDC (Arctic Environmental Information and Data Center), 1974, Alaska Regional Profiles, Southcentral Region. Arctic Environmental Information and Data Center, University of Alaska, 1974.

Barnes, D.F., 1968, Alaska gravity base station network: U.S. Geological Survey Open-File Report, $21 \mathrm{p}$.

1972, Southeast Alaska gravity base station network: U.S. Geological Survey OpenFile Report, 40 p.

1977, Bouguer gravity map of Alaska: U.S. Geological Survey Geophysical Investigations Map GP-913, scale 1:2,500,000.

Godson, R.H., and Plouff, D., 1988, BOUGUER Version 1.0, A microcomputer gravity-terraincorrection program: U.S. Geological Survey Open-File Report 88-644-A, Documentation, 22 p.; 88-644-C, 5 1/4 - inch diskette.

Hammer, S., 1939, Terrain corrections for gravimeter stations: Geophysics, v.4, p.184-194.

International Association of Geodesy, 1971, Geodetic reference system, 1967: Paris, Bureau Central de l'Association Internationale de Geodesie, Special Publication 3, 116 p. 
Jachens, R.C., and Roberts, C.W., 1981, Documentation of a FORTRAN program, 'isocomp', for computing isostatic residual gravity: U.S. Geological Survey Open-File Report 81-574, $26 \mathrm{p}$.

Kirschner, C.E., 1988, Map Showing Sedimentary Basins of Onshore and Continental Shelf Areas, Alaska: U.S. Geological Survey Miscellaneous Investigations Series Map I-1873, scale $1: 2,500,000$.

Maynard, D.L., 1987, Water-well data from the Houston Area, Matanuska-Susitna Borough, Alaska. Alaska Department of Natural Resources, Division of Geological and Geophysical Surveys, Report of Investigations 87-17.

Morelli, C., Gantar, C., Honkasala, T., McConnell, R.K., Tanner, J.G., Szabo, B., Uotila, U.A., and Whalen, C.T., 1974, The International Gravity Standardization Net 1971 (IGSN 71): Paris, Bureau Central de 1'Association Internationale de Geodesie, Special Publication 4, $194 \mathrm{p}$.

Plouff, D., 1966, Digital terrain corrections based on geographic coordinates [abs.]: Geophysics, v.31, no. 6, p. 1208 .

Plouff, D., 1977, Preliminary documentation for a Fortran program to compute gravity terrain corrections based on topography digitized on a geographic grid: U.S. Geological Survey Open-File Report 77-535, 45 p.

Ryherd, T., 2003, Personal Communication from Timothy Ryherd, Resource Evaluation Section, Division of Oil and Gas, Alaska Department of Natural Resources, October 23, 2003. 
Table 1. Principal facts for the gravity stations collected during this survey. Topo represents the topographic elevation taken from USGS topographic maps at 1:63,360 scale. Elev represents the GPS station elevation used for reducing the data. FAA is the free-air anomaly, SBA is the simple Bouguer anomaly, CBA is the complete Bouguer anomaly and IA is the isostatic anomaly.

\begin{tabular}{|c|c|c|c|c|c|c|c|c|c|c|c|}
\hline Station & Topo & & Elev & & Lat & Lon & Obs Grav & FAA & SBA & CBA & IA \\
\hline TLKN & 350.00 & $\mathrm{~F}$ & 359.00 & $\mathrm{~F}$ & 62.31681483 & -150.10045169 & 982005.714 & -55.053 & -67.297 & -67.137 & -10.927 \\
\hline S001 & 0.00 & $\mathrm{~F}$ & 419.55 & $\mathrm{~F}$ & 62.25669913 & -150.29579449 & 981981.683 & -68.897 & -83.206 & -83.296 & -33.176 \\
\hline S002 & 10 & $\mathrm{F}$ & 40976 & $\mathrm{~F}$ & 62.23502986 & -150.34035619 & 981979.009 & -70.872 & -84.847 & -84.967 & -36.307 \\
\hline S003 & 90.00 & $\mathrm{~F}$ & 381.61 & $\mathrm{~F}$ & 62.22191765 & -150.36413737 & 981980.500 & -71.047 & -84.062 & -84.192 & -36.322 \\
\hline S004 & 0.00 & $\mathrm{~F}$ & & $\mathrm{~F}$ & 62.20502818 & -150.39372319 & 981974.999 & -78.391 & -90.280 & -90.410 & -43.490 \\
\hline S005 & 45.00 & $\mathrm{~F}$ & 322.18 & $\mathrm{~F}$ & 62.19385708 & -150.41787149 & 981972.275 & -82.762 & -93.750 & -93.890 & -47.620 \\
\hline S006 & & $\mathrm{F}$ & & $\mathrm{F}$ & 62.18138800 & -150.44599100 & 981968.338 & -82.699 & -94.799 & -94.949 & -49.399 \\
\hline S007 & & $\mathrm{F}$ & 366 & $\mathrm{~F}$ & 7347327 & -150.45632174 & 981964.818 & -84.508 & -97.013 & -97.173 & -52.003 \\
\hline S008 & 370.00 & $\mathrm{~F}$ & 365.08 & $\mathrm{~F}$ & 62.16387700 & -150.47969000 & 981961.669 & -87.087 & -99.538 & -99.698 & -55.018 \\
\hline S009 & 390.00 & $\mathrm{~F}$ & 379.76 & $\mathrm{~F}$ & 62.14754223 & -150.50420059 & 981958.093 & -88.057 & -101.009 & -101.179 & -57.239 \\
\hline S010 & 00.00 & $\mathrm{~F}$ & 405.70 & $\mathrm{~F}$ & 62.13929075 & 2095008 & .879 & -88.213 & -102.050 & -102.220 & -58.650 \\
\hline S011 & 5.00 & $\mathrm{~F}$ & 406.79 & $\mathrm{~F}$ & 62.12564913 & -150.54599401 & 981950.052 & -91.915 & -105.789 & -105.949 & -63.039 \\
\hline S012 & 50 & $\mathrm{~F}$ & 47521 & $\mathrm{~F}$ & 62.11161721 & -150.57600763 & 981943.886 & -90.593 & -106.800 & -106.960 & -64.610 \\
\hline S013 & 30.00 & $\mathrm{~F}$ & 450.45 & $\mathrm{~F}$ & 62.09683769 & -150.59857261 & 3.963 & -91.735 & -107.098 & -107.238 & -65.458 \\
\hline S014 & 580.00 & $\mathrm{~F}$ & 540.67 & $\mathrm{~F}$ & 62.08668220 & -150.62289572 & 981946.298 & -80.152 & -98.592 & -98.732 & -57.332 \\
\hline S015 & 30.00 & $\mathrm{~F}$ & & $\mathrm{~F}$ & 62.05907814 & -150.67715488 & 981925.707 & -88.597 & -110.690 & & -70.410 \\
\hline S016 & 90.00 & $\mathrm{~F}$ & 689.44 & $\mathrm{~F}$ & 62.04503390 & -150.69499174 & 981920.663 & -88.666 & -112.180 & -112.330 & -72.370 \\
\hline S017 & 700.00 & $\mathrm{~F}$ & 690.34 & $\mathrm{~F}$ & 62.02892600 & -150.72119000 & 981915.651 & -92.383 & -115.928 & -116.068 & -76.658 \\
\hline S018 & & $\mathrm{F}$ & & $\mathrm{F}$ & 62.01386021 & -150.76114845 & 981915.648 & -94.915 & -117.132 & -117.272 & -78.292 \\
\hline S019 & 350.00 & $\mathrm{~F}$ & 350.55 & $\mathrm{~F}$ & 61.99722678 & -150.78902017 & 981933.492 & -104.118 & -116.074 & -116.034 & -77.484 \\
\hline S020 & 450.00 & $\mathrm{~F}$ & 413.23 & $\mathrm{~F}$ & 61.98543276 & -150.81604194 & 981926.351 & -104.476 & -118.570 & -118.740 & -80.460 \\
\hline S021 & & $\mathrm{F}$ & & $\mathrm{F}$ & 61.97364453 & -150.83598302 & & -111.948 & -120.784 & -120.814 & -82.854 \\
\hline S022 & 30.00 & $\mathrm{~F}$ & 398.71 & $\mathrm{~F}$ & 62.23411406 & -150.42136206 & 981974.272 & -76.579 & -90.177 & -90.287 & -42.237 \\
\hline S023 & 0.00 & $\mathrm{~F}$ & 544.95 & $\mathrm{~F}$ & 62.22564000 & -150.54214500 & 981957.504 & -78.958 & -97.544 & -97.614 & -50.114 \\
\hline S024 & 875.00 & $\mathrm{~F}$ & 868.60 & $\mathrm{~F}$ & 62.19689544 & -150.68067841 & 981930.126 & -73.743 & -103.367 & -103.367 & -56.777 \\
\hline S025 & 800.00 & $\mathrm{~F}$ & 788.29 & $\mathrm{~F}$ & 62.19139729 & -150.76916715 & 981935.140 & -75.870 & -102.756 & -102.286 & -55.446 \\
\hline S026 & 0.00 & $\mathrm{~F}$ & 629.94 & $\mathrm{~F}$ & 62.17854061 & -150.88201754 & 981957.705 & -67.237 & -88.721 & -88.721 & -41.681 \\
\hline S027 & 45.00 & $\mathrm{~F}$ & 951.92 & $\mathrm{~F}$ & 62.16105119 & -150.99172287 & 981934.019 & -59.327 & -91.793 & -91.773 & -44.543 \\
\hline S028 & 930.00 & $\mathrm{~F}$ & & $\mathrm{~F}$ & 62.14790241 & -150.97150657 & 981936.190 & -57.711 & -89.618 & -89.578 & -43.228 \\
\hline S029 & 90.00 & $\mathrm{~F}$ & 663.05 & $\mathrm{~F}$ & 62.13186426 & -150.95429250 & 981946.668 & -71.661 & -94.275 & -94.105 & -48.735 \\
\hline S030 & 600.00 & $\mathrm{~F}$ & 614.34 & 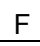 & 62.12680553 & -150.93252959 & 981945.804 & -76.727 & -97.680 & -97.400 & -52.440 \\
\hline S031 & & $\mathrm{F}$ & & 1 & 62.10800784 & -150.90788625 & 981927.987 & -84.400 & -108.520 & -108.580 & -64.710 \\
\hline S032 & 0.00 & $\mathrm{~F}$ & & $\mathrm{~F}$ & 62.08507559 & -150.88509494 & 981929.335 & -96.785 & -115.301 & -115.271 & -72.631 \\
\hline S033 & 500.00 & $\mathrm{~F}$ & 496.00 & $\mathrm{~F}$ & 62.07598498 & -150.86183121 & 981928.155 & -101.694 & -118.610 & -118.590 & -76.540 \\
\hline & & $\mathrm{F}$ & 504.10 & 1 & 62.06712513 & -150.84327944 & & -100.770 & -117.963 & -118.043 & -76.503 \\
\hline S035 & 500.00 & $\mathrm{~F}$ & 503.41 & $\mathrm{~F}$ & 62.05620500 & -150.81577200 & 981928.040 & -99.627 & -116.796 & -116.846 & -75.916 \\
\hline S036 & 460.00 & $\mathrm{~F}$ & 458.09 & 1 & 62.03612011 & -150.82296707 & 981929.287 & -101.133 & -116.757 & -116.867 & -76.707 \\
\hline S037 & 440.00 & $\mathrm{~F}$ & 431.41 & $\mathrm{~F}$ & 62.02115657 & -150.82285287 & 981929.192 & -102.613 & -117.326 & -117.426 & -77.816 \\
\hline S038 & 420.00 & $\mathrm{~F}$ & 408.25 & $\mathrm{~F}$ & 62.01107630 & -150.82484418 & 981929.282 & -103.942 & -117.866 & -117.966 & -78.726 \\
\hline
\end{tabular}


Table 1 (continued)

\begin{tabular}{|c|c|c|c|c|c|c|c|c|c|c|c|}
\hline Station & Topo & & Elev & & Lat & Lon & Obs Grav & FAA & SBA & CBA & IA \\
\hline S039 & 345.00 & $\mathrm{~F}$ & & $\mathrm{~F}$ & 61.98502440 & -150.77566955 & 981933.422 & -105.090 & -116.386 & -116.466 & -78.466 \\
\hline S040 & 500 & $\mathrm{~F}$ & 286.09 & $\mathrm{~F}$ & 1.97814850 & -150.74865657 & 981934.616 & -107.622 & -117.379 & -117.139 & -79.499 \\
\hline S041 & & $\mathrm{F}$ & 200.00 & $\mathrm{~F}$ & 61.96333603 & -150.73375026 & & -111.366 & -120.563 & -120.553 & -83.483 \\
\hline S042 & & $\mathrm{F}$ & & $\mathrm{F}$ & & & & & & -120.869 & 329 \\
\hline S043 & 90.00 & $\mathrm{~F}$ & 191.57 & $\mathrm{~F}$ & 61.93495618 & -150.70601132 & 981935.792 & -112.085 & -118.618 & -118.618 & -82.498 \\
\hline S044 & (2) & $\mathrm{F}$ & & $\mathrm{F}$ & 61.92490425 & -150.69413944 & 081937909 & -111.195 & -117.009 & -117.079 & -81.319 \\
\hline S045 & & $\mathrm{F}$ & & $\mathrm{F}$ & & & & -110.298 & -115.601 & -115.691 & -80.311 \\
\hline S046 & & $\mathrm{F}$ & & $\mathrm{F}$ & 61.89535292 & -150.66818870 & & -106.550 & -111.620 & & -76.830 \\
\hline S047 & & $\mathrm{F}$ & 406.99 & $\mathrm{~F}$ & 61.87202453 & -150.56514050 & 981945.008 & -77.862 & -91.743 & -91.973 & -57.743 \\
\hline S048 & & $\mathrm{F}$ & & $\mathrm{F}$ & 61.85511009 & -150.53462809 &.$<30$ & -85.402 & -96.730 & -96.970 & -63.090 \\
\hline S049 & & $\mathrm{F}$ & & $\mathrm{F}$ & 941 & 237 & & & .756 & .996 & .266 \\
\hline S050 & 25.00 & $\mathrm{~F}$ & 294.13 & $\mathrm{~F}$ & 61.83261642 & -150.48695760 & 981947.519 & -82.991 & -93.023 & -93.273 & -59.803 \\
\hline S051 & 0.00 & $\mathrm{~F}$ & 257.77 & $\mathrm{~F}$ & 61.81656058 & -150.46863425 & 901040.042 & -83.175 & -91.967 & -92.227 & -59.047 \\
\hline S052 & 5.00 & $\mathrm{~F}$ & & $\mathrm{~F}$ & 263 & 546 & 977 & -84.820 & -92.155 & -92.415 & 9.365 \\
\hline S053 & & $\mathrm{F}$ & 204.00 & $\mathrm{~F}$ & 61.79277652 & -150.42443138 & 582 & .396 & .353 & -92.613 & -59.703 \\
\hline S054 & 30.00 & $\mathrm{~F}$ & 193.13 & $\mathrm{~F}$ & 61.77868378 & -150.40561968 & 981951.037 & -84.898 & -91.485 & -91.745 & -59.045 \\
\hline S055 & 90.00 & $\mathrm{~F}$ & 173.01 & $\mathrm{~F}$ & 61.76720354 & -150.38641498 & 981900.100 & -86.137 & -92.058 & -92.318 & -59.728 \\
\hline S056 & 370.00 & $\mathrm{~F}$ & & $\mathrm{~F}$ & 62.24718520 & -150.19039937 & & -64.982 & -77.725 & -77.755 & .335 \\
\hline S057 & & $\mathrm{F}$ & & $\mathrm{F}$ & & & & & & & \\
\hline S058 & & $\mathrm{F}$ & & $\mathrm{F}$ & & & & -77.060 & 658 & -88.818 & .538 \\
\hline S059 & & $\mathrm{F}$ & & $\mathrm{F}$ & 0649954 & 2371 & .198 & -86.541 & 5.626 & -95.836 & .846 \\
\hline S060 & & $\mathrm{F}$ & & $\mathrm{F}$ & 62.09956538 & -150.25652294 & & -75.977 & -87.334 & -87.484 & -42.924 \\
\hline S061 & & $\mathrm{F}$ & 351.64 & $\mathrm{~F}$ & 62.13852785 & -150.23430236 & 901911.315 & -70.745 & -82.738 & -82.868 & -36.468 \\
\hline S062 & & $\mathrm{F}$ & 328.06 & $\mathrm{~F}$ & 62.10518985 & 2585985 & 981953.655 & -94.183 & -105.371 & -105.551 & 3.441 \\
\hline S063 & ل & $\mathrm{F}$ & 289.42 & $\mathrm{~F}$ & 62.07109774 & -150.51072747 & 981952.555 & -96.357 & -106.228 & -106.438 & -65.588 \\
\hline S064 & & $\mathrm{F}$ & & $\mathrm{F}$ & 62.00026737 & -150.46543947 & 981953.092 & -95.229 & -103.384 & -103.614 & -65.064 \\
\hline S065 & 0.00 & $\mathrm{~F}$ & 198.63 & $\mathrm{~F}$ & 61.96441512 & -150.45676347 & 981953.738 & -95.692 & -102.467 & -102.727 & 5.347 \\
\hline S066 & 90.00 & $\mathrm{~F}$ & 181.69 & $\mathrm{~F}$ & 61.92946209 & -150.45746494 & 981954.213 & -94.178 & -100.375 & -100.625 & -64.355 \\
\hline S067 & & $\mathrm{F}$ & & $\mathrm{F}$ & & & & & -96.968 & -97.218 & -61.868 \\
\hline S068 & 190.00 & $\mathrm{~F}$ & & $\mathrm{~F}$ & 61.85875519 & -150.42655976 & 981956.348 & -84.541 & -91.525 & -91.785 & -57.275 \\
\hline S069 & 40.00 & $\mathrm{~F}$ & 105.97 & $F$ & 61.85719800 & -150.36887100 & 981959.122 & -90.942 & -94.556 & -94.816 & -59.806 \\
\hline & & $\mathrm{F}$ & & & & -150.34775006 & & & & & -55.556 \\
\hline S071 & .00 & $\mathrm{~F}$ & 122.96 & $\mathrm{~F}$ & 61.82036782 & -150.19482413 & 981976.338 & -69.348 & -73.541 & -73.711 & -36.791 \\
\hline S072 & 5.00 & $\mathrm{~F}$ & 144.46 & $\mathrm{~F}$ & 61.87778677 & -150.15899046 & 981970.906 & -77.091 & -82.018 & -82.128 & -42.568 \\
\hline S073 & & $\mathrm{F}$ & & $r$ & 61.93727581 & -150.18895073 & 981975.744 & -73.827 & -79.810 & -79.940 & -39.260 \\
\hline S074 & & $\mathrm{F}$ & & $\mathrm{r}$ & 61.98073400 & -150.14547000 & 981981.204 & -71.999 & -77.851 & -77.911 & -34.641 \\
\hline S075 & & $\mathrm{F}$ & & $\mathrm{F}$ & 61.95877810 & -150.84955455 & 981931.650 & -117.974 & -124.524 & -124.684 & -87.114 \\
\hline S076 & 150.00 & $\mathrm{~F}$ & 155.60 & 5 & 61.94801987 & -150.87536270 & 981931.745 & -120.499 & -125.806 & -126.006 & -88.546 \\
\hline S077 & 150.00 & $\mathrm{~F}$ & 141.22 & $\Gamma$ & 61.93524410 & -150.90699940 & 981930.138 & -122.495 & -127.312 & -127.532 & -90.152 \\
\hline S078 & 140.00 & $\mathrm{~F}$ & 4.51 & $\mathrm{~F}$ & 61.89597700 & -150.95193500 & 981929.285 & -122.895 & -126.803 & -127.003 & -90.083 \\
\hline & & $\mathrm{F}$ & & 1 & 61.89686359 & -150.99163567 & 981919.608 & -118.160 & -127.318 & -127.508 & -90.038 \\
\hline S080 & 290.00 & $\mathrm{~F}$ & 305.65 & $\mathrm{~F}$ & 61.88279501 & -150.99974397 & 981915.584 & -117.629 & -128.054 & -128.244 & -90.894 \\
\hline S081 & 320.00 & $\mathrm{~F}$ & 348.78 & $\mathrm{r}$ & 61.87130285 & -151.02582580 & 981913.338 & -114.952 & -126.848 & -127.028 & -89.488 \\
\hline
\end{tabular}


Table 1 (continued)

\begin{tabular}{|c|c|c|c|c|c|c|c|c|c|c|c|}
\hline Station & opo & & Elev & & Lat & Lon & Obs Grav & FAA & SBA & CBA & IA \\
\hline S082 & 250.00 & $\mathrm{~F}$ & 281.47 & $\mathrm{~F}$ & 61.85888706 & -151.04562581 & 981918.023 & -115.661 & -125.261 & -125.431 & -87.711 \\
\hline S083 & 30 & $\mathrm{~F}$ & 202.73 & $\mathrm{~F}$ & 61.84721706 & -151.06747276 & 981923964 & -116.245 & -123.160 & -123.300 & -85.310 \\
\hline S084 & & $\mathrm{F}$ & & $\mathrm{F}$ & 61.84272158 & -151.08364543 & & -115.471 & -122.714 & -122.844 & -84.594 \\
\hline S085 & & $\mathrm{F}$ & & $\mathrm{F}$ & 61.83115596 & & & -118.356 & & & -85.992 \\
\hline S086 & 80.00 & $\mathrm{~F}$ & 219.18 & $\mathrm{~F}$ & 61.81742400 & -151.12625500 & 981920501 & -115.912 & -123.387 & -123.377 & -84.367 \\
\hline S087 & & $\mathrm{F}$ & & $\mathrm{F}$ & 61.79135029 & -151.16996015 & & -102.743 & -112.082 & -111.622 & -71.602 \\
\hline S088 & & $\mathrm{F}$ & & $\mathrm{F}$ & 23748 & -151.1985 & & 91.535 & -104.959 & -102.959 & -62.159 \\
\hline S089 & 80.00 & $\mathrm{~F}$ & 271.84 & $\mathrm{~F}$ & 61.84881020 & -151.35687048 & 981968.542 & -65.287 & -74.559 & -74.269 & -29.719 \\
\hline S090 & & $\mathrm{F}$ & 238.76 & $\mathrm{~F}$ & 61.86478888 & -151.41800872 & 981970.533 & -67.614 & -75.757 & -75.467 & -29.267 \\
\hline S091 & 0.00 & $\mathrm{~F}$ & 220 & $\mathrm{~F}$ & 61.87985200 & .34222600 & .140 & -70.892 & -78.401 & -78.261 & 4.261 \\
\hline S092 & 5.00 & $\mathrm{~F}$ & 190.11 & $\mathrm{~F}$ & 61.93493000 & -151.31711300 & & -81.640 & -88.124 & -88.134 & -44.564 \\
\hline S093 & & $\mathrm{F}$ & 157.72 & $\mathrm{~F}$ & 61.95506529 & -151.25769884 & 981971.717 & -80.858 & -86.237 & -86.287 & -43.657 \\
\hline S094 & 60.00 & $\mathrm{~F}$ & 145.05 & $\mathrm{~F}$ & 61.96600871 & -151.18394195 & .428 & -98.163 & -103.110 & -103.160 & -61.560 \\
\hline S095 & 5.00 & $\mathrm{~F}$ & 196.86 & $\mathrm{~F}$ & 61.97037078 & -150.91473747 & 932.274 & -117.772 & -124.486 & -124.606 & -86.146 \\
\hline S096 & 350.00 & $\mathrm{~F}$ & 288.96 & $\mathrm{~F}$ & 62.20762721 & -150.15835213 & 981982.701 & -76.491 & -86.346 & -86.366 & -35.756 \\
\hline S097 & 40.00 & $\mathrm{~F}$ & 241.65 & $\mathrm{~F}$ & 62.12083600 & -150.10903000 & 981991.609 & -65.530 & -73.772 & -73.742 & -24.792 \\
\hline S098 & 10.00 & $\mathrm{~F}$ & 305.61 & $\mathrm{~F}$ & 62.05743062 & -150.24179760 & 981966.860 & -79.503 & -89.927 & -90.087 & -46.687 \\
\hline S099 & 190.00 & $\mathrm{~F}$ & 184.80 & $\mathrm{~F}$ & 62.03349486 & -150.29897860 & 981968.300 & -87.627 & -93.930 & -94.110 & -52.580 \\
\hline $\mathrm{S} 100$ & 30.00 & $\mathrm{~F}$ & 219.37 & $\mathrm{~F}$ & 62.00091900 & -150.37335400 & .903 & -91.324 & -98.806 & -99.036 & -59.636 \\
\hline S101 & 5.00 & $\mathrm{~F}$ & & $\mathrm{~F}$ & 56916 & -150.40844662 & 171 & .439 & -99.973 & .213 & .733 \\
\hline S102 & 0.00 & $\mathrm{~F}$ & 324.43 & $\mathrm{~F}$ & 61.92905884 & -150.53361508 & 981941 & -93.065 & -104.130 & -104.370 & -68.480 \\
\hline S103 & 10.00 & $\mathrm{~F}$ & & $\mathrm{~F}$ & 61.89175584 & -150.60801903 & 981931.018 & -91.297 & -105.918 & -106.138 & -71.388 \\
\hline S104 & 0.00 & $\mathrm{~F}$ & & $\mathrm{~F}$ & 61.86214080 & -150.65244869 & 981939.826 & -100.683 & -107.897 & -108.147 & 4.117 \\
\hline S105 & 130.00 & $\mathrm{~F}$ & 155.63 & $\mathrm{~F}$ & 61.84746653 & -150.71892705 & 981931.791 & -112.867 & -118.175 & -118.465 & -84.515 \\
\hline S106 & 0.00 & $\mathrm{~F}$ & & $\mathrm{~F}$ & 61.82233400 & -150.75313300 & 981929.132 & -118.651 & -122.138 & -122.418 & -88.728 \\
\hline S107 & 120.00 & $\mathrm{~F}$ & 179.53 & $\mathrm{~F}$ & 61.79755300 & -150.82025800 & 981916.363 & -122.278 & -128.401 & -128.641 & -94.671 \\
\hline $\mathrm{S} 108$ & 140.00 & $\mathrm{~F}$ & 150.73 & $\mathrm{~F}$ & 61.76859868 & -150.90018615 & 981916.677 & -122.484 & -127.625 & -127.805 & -93.035 \\
\hline & & $\mathrm{F}$ & & $\mathrm{F}$ & & -150.90292638 & & -121.201 & -127.685 & -127.895 & -92.835 \\
\hline $\mathrm{S} 110$ & 150.00 & $\mathrm{~F}$ & 192.03 & $\mathrm{~F}$ & 61.83728436 & -150.88839100 & 981918.601 & -121.866 & -128.415 & -128.605 & -93.385 \\
\hline S111 & 100.00 & $\mathrm{~F}$ & 80.11 & $\mathrm{~F}$ & 61.86384195 & -150.86755625 & 981927.750 & -125.248 & -127.980 & -128.230 & -92.850 \\
\hline & & $\mathrm{F}$ & & & & -150.81828800 & & -122.011 & -125.089 & & -90.349 \\
\hline $\mathrm{S} 113$ & 120.00 & $\mathrm{~F}$ & 117.29 & $\mathrm{~F}$ & 61.89769304 & -150.77617608 & 981933.356 & -118.699 & -122.699 & -122.959 & -87.599 \\
\hline S114 & 200.00 & $\mathrm{~F}$ & 188.17 & $\mathrm{~F}$ & 61.92955278 & -150.78613000 & 981932.373 & -115.416 & -121.834 & -122.064 & -85.794 \\
\hline S115 & 300.00 & $\mathrm{~F}$ & & 1 & 61.95539693 & -150.77788258 & 981929.529 & -111.304 & -120.950 & -121.130 & -84.110 \\
\hline S116 & 170.00 & $\mathrm{~F}$ & 174.57 & F & 61.93922600 & -151.29253400 & 981966.457 & -83.341 & -89.294 & -89.344 & -46.244 \\
\hline & 180.00 & $\mathrm{~F}$ & 174.18 & 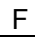 & 61.91415715 & -151.22880229 & 981963.416 & -84.528 & -90.469 & -90.569 & -49.049 \\
\hline S118 & 190.00 & $\mathrm{~F}$ & 179.76 & $\mathrm{~F}$ & 61.86612782 & -151.13313687 & 981924.493 & -119.304 & -125.435 & -125.545 & -86.255 \\
\hline $\mathrm{S} 119$ & 195.00 & $\mathrm{~F}$ & 191.28 & $\mathrm{~F}$ & 61.81733474 & -151.02125501 & 981920.551 & -118.479 & -125.003 & -125.143 & -88.143 \\
\hline $\mathrm{S} 120$ & 185.00 & $\mathrm{~F}$ & 159.27 & $\mathrm{~F}$ & 61.79707475 & -150.97183437 & 981919.072 & -121.438 & -126.870 & -127.010 & -90.960 \\
\hline
\end{tabular}




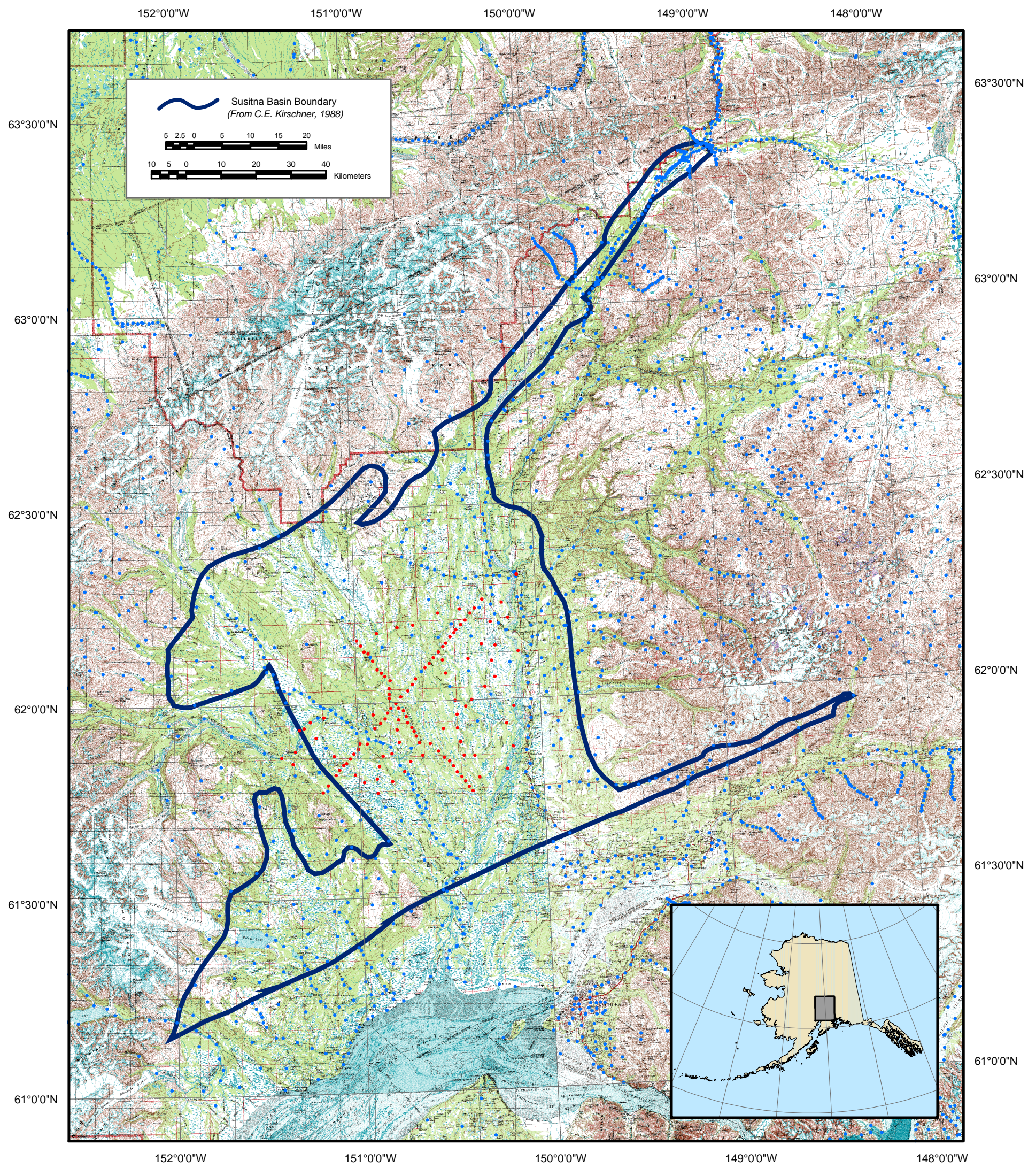

Figure 2. Index map of the study area showing the locations of the gravity data collected for this study in red. The previously collected data is shown in black. The map base is a composite of USGS 1:250,000 scale topographic maps. 


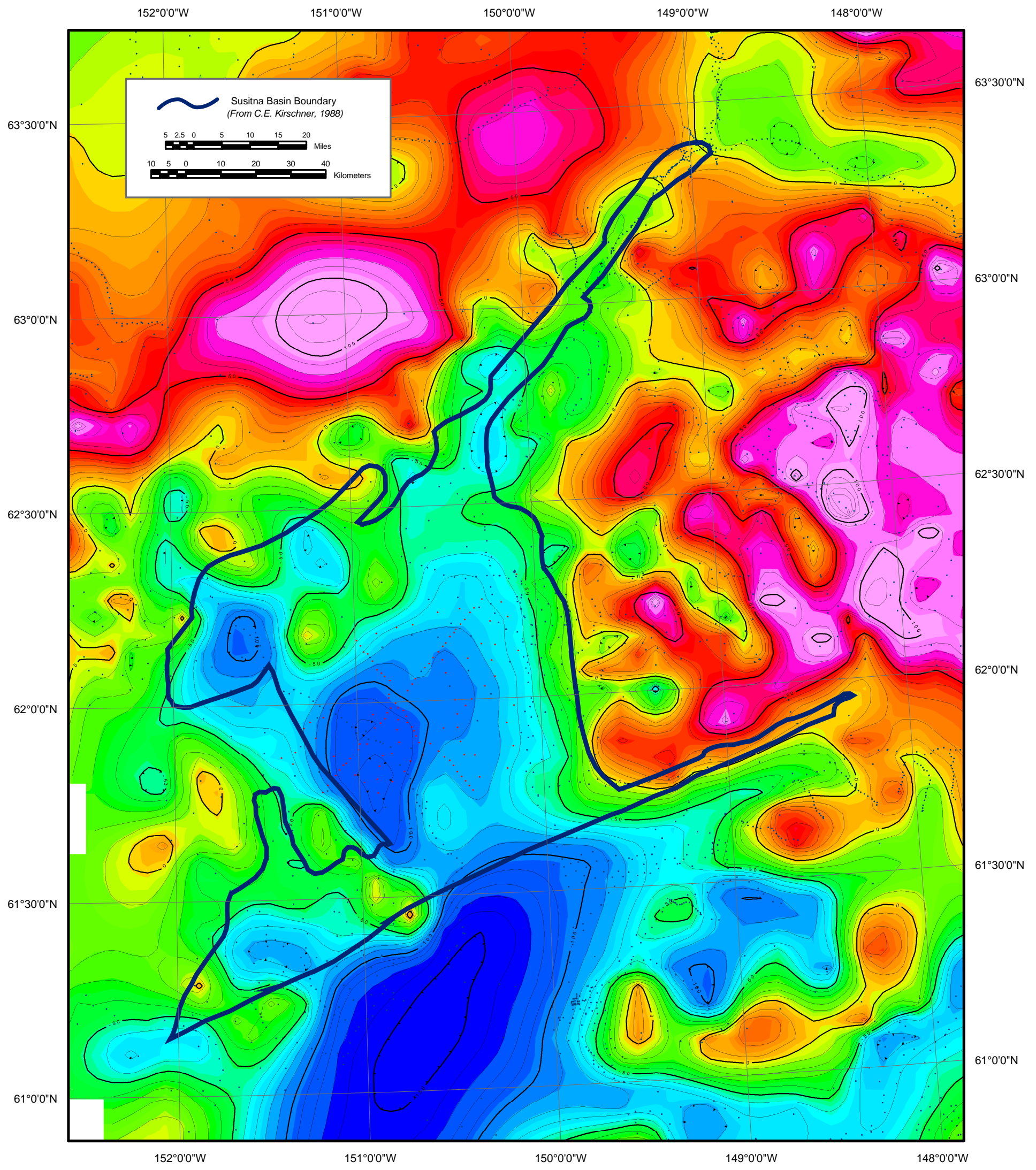

Figure 3. Free-air gravity map of the Susitna Basin with a contour interval of $10 \mathrm{mGal}$. 


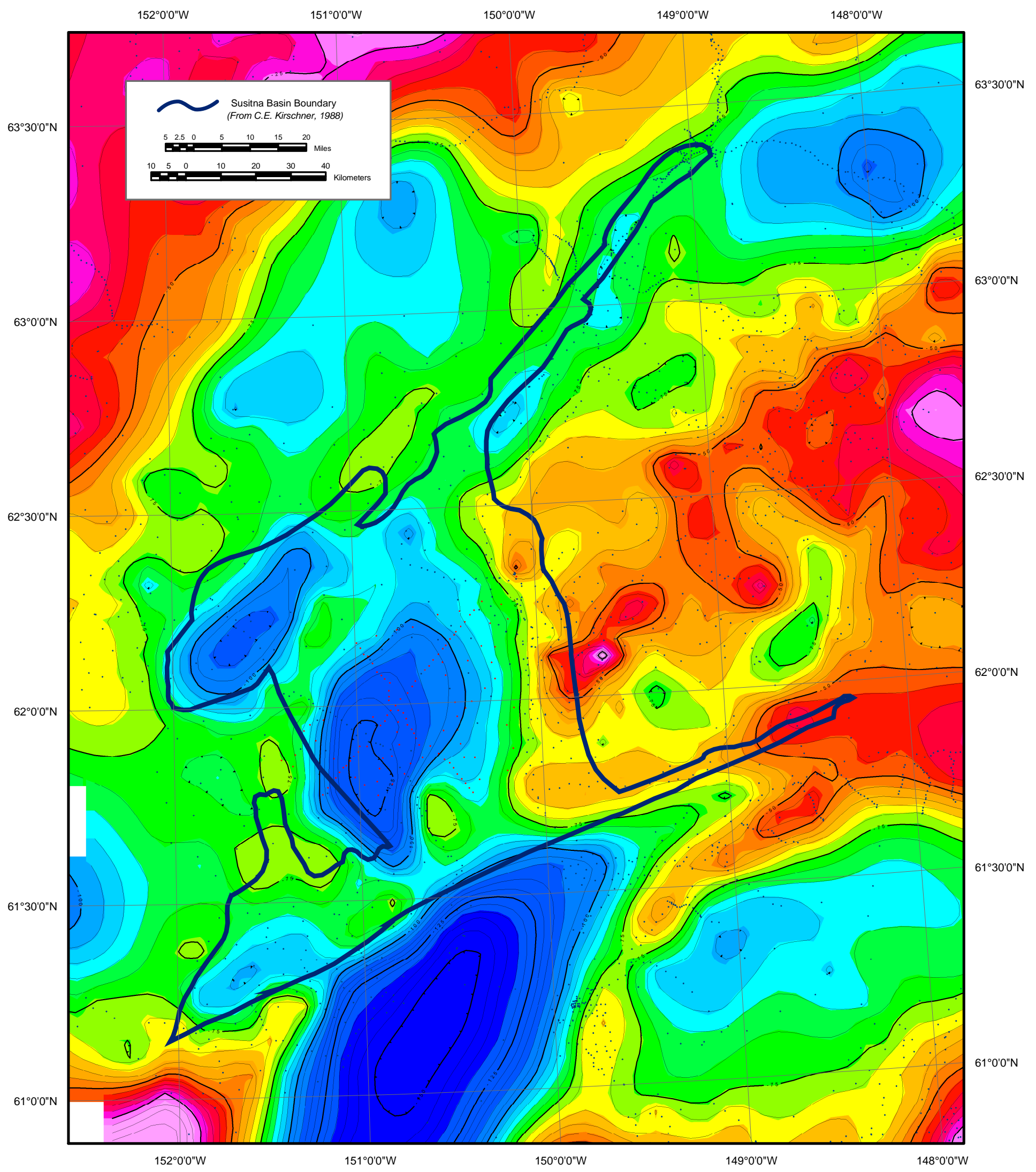

Figure 4. Complete Bouguer gravity map of the Susitna Basin with a contour interval of $5 \mathrm{mGal}$. 


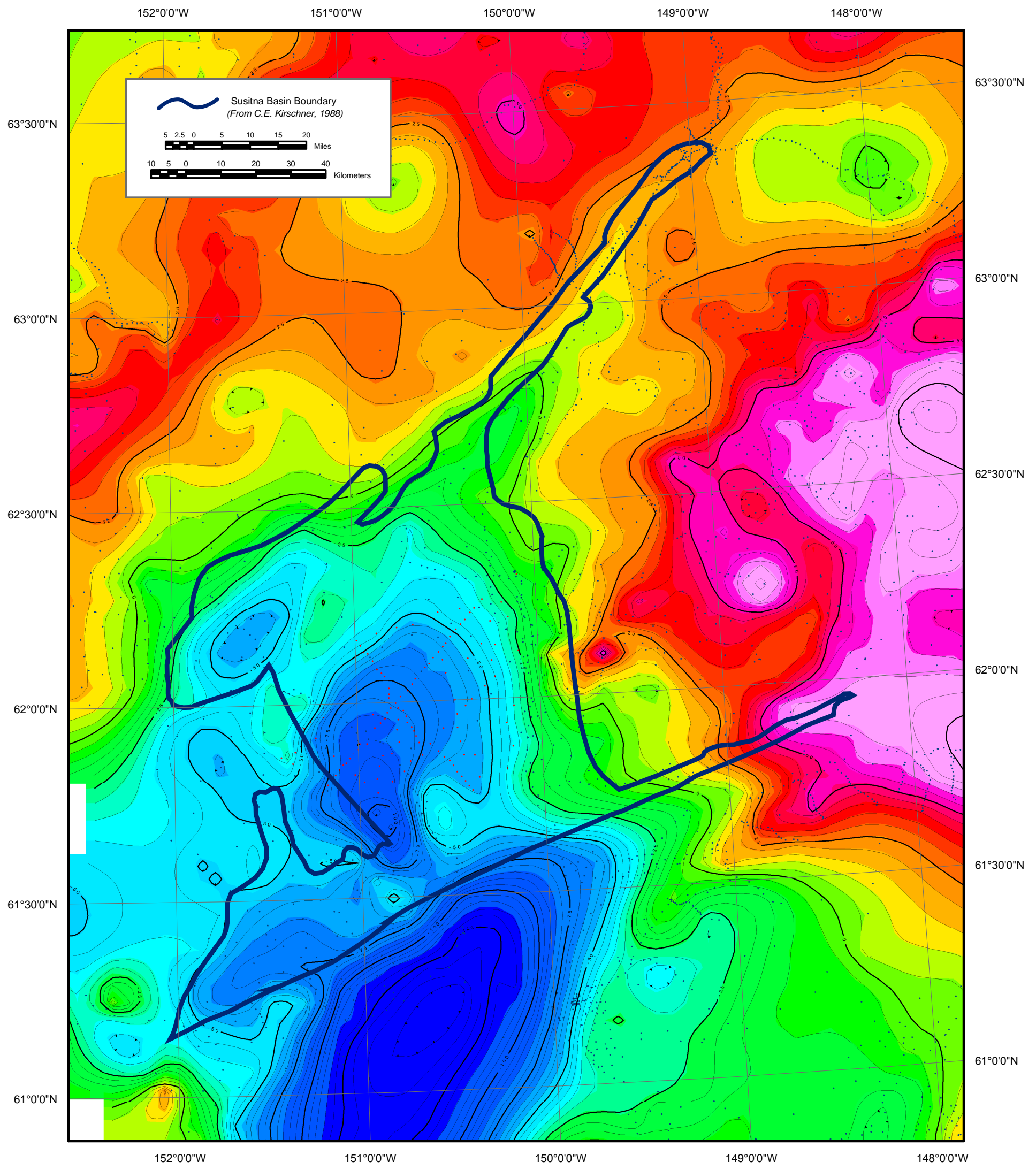

Figure 5. Isostatic gravity map of the Susitna Basin with a contour interval of $5 \mathrm{mGal}$. 